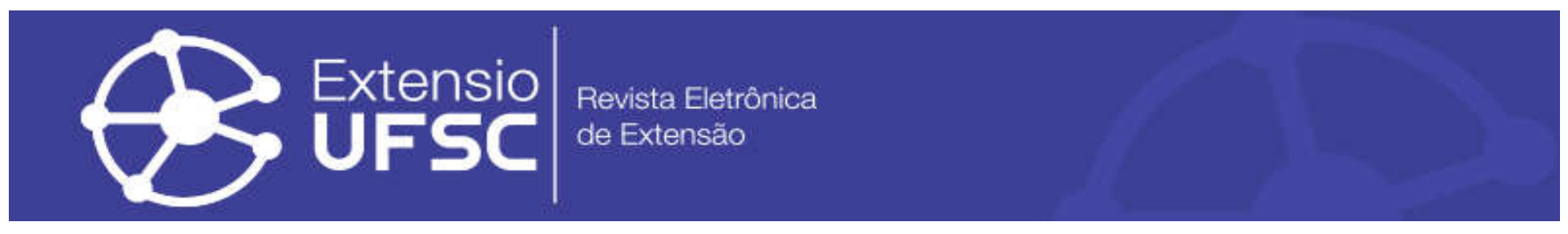

\title{
PREVENÇÃO AO CÂNCER DE PELE EM DISCENTES DO CURSO DE ZOOTECNIA: UM RELATO DE EXPERIÊNCIA
}

\author{
Isabela Leopoldino Dourado \\ Faculdade Evangélica de Ceres \\ douradoisabela29@gmail.com \\ Luiz Augusto Vieira Caetano \\ Faculdade Evangélica de Ceres \\ vieiraaugustocaetano1910@gmail.com \\ Carla Danielle Dias Costa \\ Centro Universitário de Mineiros \\ carladaniellebm@gmail.com
}

\section{Resumo}

O câncer de pele é uma dermatose comum entre os seres humanos, sendo de etiologia multifatorial, na qual são considerados fatores como predisposição genética, exposição a fatores ambientais de risco e outros. Dentre a classe trabalhadora que apresenta grande risco em desenvolver o câncer de pele, estão os trabalhadores rurais, pois estes passam um longo período exposto às radiações solares. $\mathrm{O}$ objetivo deste trabalho foi levar informações referente à prevenção ao desenvolvimento do câncer de pele voltado a alunos do curso de Zootecnia, por meio de atividades que informavam as maneiras adequadas de exercerem suas atividades acadêmicas com segurança, uma vez que estão mais expostos aos raios solares devido às suas práticas acadêmicas. Trata-se de um relato de experiência desenvolvido com alunos do primeiro período do curso de Zootecnia de uma instituição pública da cidade de Ceres no estado de Goiás, entre os anos de 2018 e 2019. As atividades foram desenvolvidas visando abordar temas relacionados ao câncer de pele e à fotoproteção. Foi possível identificar que os alunos possuíam baixo conhecimento sobre tema. Tal padrão de conhecimento pode ser justificado pelo fato de os mesmos estarem cursando o primeiro período do curso de Zootecnia, etapa na qual são ministradas disciplinas básicas como introdução a zootecnia, metodologia científica, anatomia animal, em que são trabalhados conteúdos relacionados ao tema. Através desta atividade extensionista, foi possível obter uma experiência acadêmica de extrema relevância e tentar gerar conscientização sobre o câncer de pele e a importância da fotoproteção. Palavras-chave: Zootecnista. Proteção Solar. Câncer de Pele. Universitário.

\section{SKIN CANCER PREVENTION IN ZOOTECHNICS STUDENTS: AN EXPERIENCE REPORT}

\begin{abstract}
Skin cancer is a common dermatosis among humans, being of multifactorial etiology, in which factors such as genetic predisposition, exposure to environmental risk factors and others are considered. Among the working class that is at high risk for developing skin cancer are rural workers, as they spend a long time exposed to solar radiation. The aim of this paper was to provide information regarding prevention of skin cancer development aimed at students of the Zootechnics course, through activities that informed the appropriate ways to perform their academic activities safely, since they are more exposed to sunlight due to their academic practices. This is an experience report developed with students of the first period of the Animal Science course of a public institution in the city of Ceres, in the state of Goiás, between 2018 and 2019. The activities were developed to address topics related to skin cancer and photoprotection. It was possible to identify that the students had low knowledge about the subject. Such pattern of knowledge can be justified by the fact that they are attending the first period of the Zootechnics course, stage in which basic subjects are taught as introduction to zootechnics, scientific methodology, animal anatomy, in which content related to the subject is not worked. Through this extension activity, it was possible to obtain an extremely relevant academic experience and try to raise awareness about skin cancer and the importance of photoprotection.
\end{abstract}

Keywords: Zootechnist. Sun Protection. Skin Cancer. University Student. 
Prevenção ao câncer de pele em discentes do curso de Zootecnia: um relato de experiência

\title{
PREVENCIÓN DEL CÁNCER DE PIEL EN ESTUDIANTES DE ZOOTECNIA: UN INFORME DE EXPERIENCIA
}

\begin{abstract}
Resumen
El cáncer de piel es una dermatosis común entre los humanos, siendo de etiología multifactorial, en la que se consideran factores como la predisposición genética, la exposición a factores de riesgo ambientales y otros. Entre la clase trabajadora que está en alto riesgo de desarrollar cáncer de piel se encuentran los trabajadores rurales, ya que pasan mucho tiempo expuestos a la radiación solar. El objetivo de este documento fue proporcionar información sobre la prevención del desarrollo del cáncer de piel dirigida a los estudiantes del curso de Zootecnia, a través de actividades que informaran las formas adecuadas de llevar a cabo sus actividades académicas de forma segura, ya que están más expuestos a la luz solar debido a sus prácticas académicas. Este es un informe de experiencia desarrollado con estudiantes del primer período del curso de Zootecnia de una institución pública en la ciudad de Ceres en el estado de Goiás, entre 2018 y 2019. Las actividades se desarrollaron para abordar temas relacionados con el cáncer de piel y la fotoprotección. Fue posible identificar que los estudiantes tenían poco conocimiento sobre el tema. Tal patrón de conocimiento puede estar justificado por el hecho de que están asistiendo al primer período del curso de Zootecnia, una etapa en la que se enseñan disciplinas básicas como la introducción a la zootecnia, la metodología científica, la anatomía animal, en la que no se trabaja el contenido relacionado con el tema. A través de esta actividad de extensión, fue posible obtener una experiencia académica extremadamente relevante e intentar crear conciencia sobre el cáncer de piel y la importancia de la fotoprotección. Palabras clave: Zootecnista. Protección Solar. Cáncer de Piel. Estudiante Universitario.
\end{abstract}


Prevenção ao câncer de pele em discentes do curso de Zootecnia: um relato de experiência

\section{INTRODUÇÃO}

O câncer de pele é uma dermatose comum entre os seres humanos, sendo de etiologia multifatorial, na qual são considerados fatores como predisposição genética, exposição a fatores ambientais de risco e outros (TRICHOPOULOS, LI, HUNTER, 1996). Atualmente no mundo ele é caracterizado como a neoplasia mais comum entre os caucasianos e pode ser classificado de duas formas: como melanoma e não melanoma (BRASIL, 2018).

No Brasil o câncer, tipo melanoma, atinge cerca de 8.450, sendo 4.200 homens e 4.250 mulheres (BRASIL, 2021). Ele tem origem a partir dos melanócitos, células que se localizam na camada basal da epiderme e têm a função de sintetizar melanina, o pigmento que determina a cor da pele. Apesar do tipo melanoma corresponder a apenas 3\% dos casos, é o mais agressivo e é responsável por 75\% das mortes por câncer de pele na população brasileira (MOHER,2014).

O câncer de pele não melanoma é o mais prevalente no Brasil e corresponde a cerca de $30 \%$ de todos os tumores malignos registrados no país. Atualmente ele acomete cerca de 176.930, sendo 83.770 homens e 93.160 mulheres, no entanto apresenta altos percentuais de cura e menor taxa de mortalidade se for detectado e tratado precocemente. No entanto, caso não seja tratado adequadamente pode deixar mutilações bastante expressivas (BRASIL, 2021).

Diversos fatores são responsáveis pelo desenvolvimento desta doença, como a cor da pele, horário e tempo de exposição, uso de imunossupressores crônicos e residência em países tropicais (BRASIL, 2014). A descendência do indivíduo, a cor do cabelo, possuir olhos claros, sardas, ser ruivo, com ancestrais célticos e fototipo de Fitzpatrick I e II garantem risco elevado para o desenvolvimento do câncer de pele não melanoma. Também são consideradas características preocupantes a tendência a queimaduras, bronzeamento com exposição solar e história de queimaduras solares na infância. Isso ocorre porque a melanina desempenha um efeito protetor na pele por dificultar a penetração dos raios solares (ZINK, 2014).

Os protetores solares ou fotoprotetores são produtos elaborados a partir da conjugação dos filtros solares, considerados ingredientes ativos, com veículos como loções hidroalcoólicas, óleos, aerossóis, cremes, emulsões e géis. A partir dessa associação formam-se diversos compostos finais, com diferentes espectros de ação contra a radiação solar, textura e fotoestabilidade, entre outros aspectos. (FLOR; DAVOLOS; CORREA, 2007).

Em 2016, a Sociedade Brasileira de Dermatologia (SBD) destacou alguns hábitos da população brasileira, que se manifestavam na exposição solar intencional e desprotegida, aumentando o risco de câncer de pele. A SBD observou também que a orientação correta é 
Prevenção ao câncer de pele em discentes do curso de Zootecnia: um relato de experiência

muito importante, pois determina os cuidados e os comportamentos que devem ser tomados para minimizar os efeitos da exposição. (Sociedade Brasileira de Dermatologia [SBD], 2020).

As profissões com maior predomínio de exposição solar são aquelas cujas atividades são exercidas principalmente em ambiente externo e aberto, como: agricultor, motorista, pedreiro (construtor), jardineiro, lavrador, carpinteiro e vigilante. (PURIM et al., 2007). Os trabalhadores rurais apresentam grande chance de desenvolver o câncer de pele, pois estes passam um longo período exposto às radiações solares, chegando a ser o principal fator de risco para cerca de $90 \%$ dos cânceres de pele do tipo não melanoma. Dependendo da região em que atua, o trabalhador acaba se expondo mais ao sol devido a estar próximo à região equatorial e não se proteger adequadamente (DALCIN, 2021).

Tem-se como importância orientar jovens ainda em sua formação acadêmica nas áreas de ciências agrárias sobre os riscos à saúde e a precaução contra o câncer de pele, porque é nessa faixa etária que estes indivíduos permanecem grande parte do tempo ao ar livre (COSTA; WEBER, 2004).

O objetivo deste trabalho é relatar a experiência de promoção a prevenção ao desenvolvimento do câncer de pele voltado a alunos do curso de Zootecnia, por meio de atividades que levaram informações de maneiras adequadas de exercerem suas atividades acadêmicas com segurança, uma vez que estão mais expostos aos raios solares devido às suas práticas acadêmicas.

\section{MATERIAIS E MÉTODOS}

O presente trabalho trata-se de um relato de experiência, que foi desenvolvido por uma discente do quarto período do curso de Biomedicina da Faculdade Evangélica de Ceres, supervisionada pela docente coordenadora do projeto. As atividades foram realizadas com alunos do primeiro período do curso de graduação de Zootecnia de um Instituto Federal da cidade de Ceres no estado de Goiás.

Com o intuito de levar informações aos estudantes sobre o uso de protetores solares em suas aulas práticas, foram planejadas atividades durante os anos de 2018 e 2019, de modo que a intervenção foi realizada em julho de 2019. O planejamento constituiu-se de três processos: revisão da literatura, elaboração das atividades e intervenção. 
Prevenção ao câncer de pele em discentes do curso de Zootecnia: um relato de experiência

\section{Etapa 1: Revisão da literatura}

Durante o processo de revisão da bibliográfica foram feitas pesquisas em bancos de dados, visando aproximação e aprofundamento com os assuntos abordados, como o câncer de pele melanoma, não melanoma e o uso de fotoproteção. Tal etapa gerou maior conhecimento sobre o tema e sobre as metodologias a serem trabalhadas, promovendo maior segurança acadêmica para realização deste trabalho.

\section{Etapa 2: Desenvolvimento do planejamento das atividades a serem realizadas com os estudantes}

Nesta etapa buscaram-se atividades que fossem didáticas e propiciassem a participação e envolvimentos dos alunos com a pesquisadora.

Para a execução da intervenção na instituição de ensino, foi realizado contato prévio com o coordenador do curso de Zootecnia, no qual foi explicado o objetivo do trabalho e solicitada a autorização para realização da atividade. Após a autorização do mesmo, os pesquisadores foram orientados quanto ao dia e ao horário adequado para a execução da ação, de modo que não interferisse ou prejudicasse o horário de aula dos discentes.

A atividade teve uma duração de cerca de sessenta minutos, de forma que foi realizada uma dinâmica com cartões $\mathrm{QR}$ code e palestra informativa, em um único dia, no período vespertino.

\section{Etapa 3: Intervenção sobre câncer de pele, uso de protetores solares e fotoproteção}

No dia determinado, a discente acompanhada pela coordenadora do projeto apresentouse à turma informando brevemente do que se tratava o projeto, os seus objetivos e explicando como aconteceriam as atividades.

Primeiramente foi aplicada uma dinâmica com o aplicativo Plickers, que se baseia em uma ferramenta, disponível na versão web e disponível para dispositivos móveis, os quais permitem aplicação de testes rápidos, que possibilitam o professor escanear as respostas e conhecer em tempo real o nível da turma quanto ao entendimento de conceitos e pontos chaves de uma aula. A ferramenta gera e salva automaticamente o desempenho individual dos alunos, criando gráficos e dados. A ferramenta Plickers, facilita e melhora a avaliação diagnóstica e formativa dos conteúdos que foram abordados (DE OLIVEIRA et al., 2018).

Cada aluno que estava presente recebeu um cartão resposta, no qual tinha um QR code para as alternativas A, B, C e D (Figura 1). Algumas questões gerais foram elaboradas sobre câncer de pele, frequência diária do uso de protetor solar, histórico de câncer de pele na família, 
conhecimento sobre fotoproteção. Tais perguntas foram direcionadas aos discentes, para que os mesmos as respondessem de acordo com o seu conhecimento sobre o tema, utilizando o cartão resposta. Todos os cartões foram scaneados após cada pergunta, permitindo identificar os padrões de respostas.

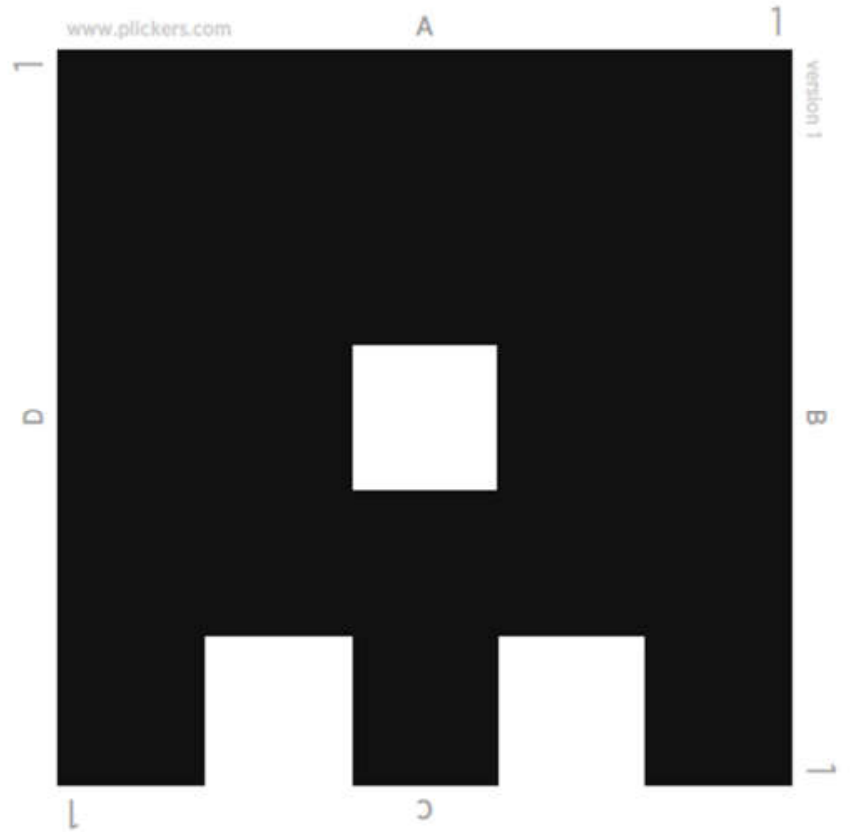

Figura 1: Padrão de cartão respostas, desenvolvido pela ferramenta Plickers que foi entregue para os discentes do primeiro período do curso de Zootecnia, a fim de verificar o conhecimento sobre o câncer de pele, uso de protetores solares e fotoproteção.

Posteriormente à dinâmica, realizou-se uma palestra projetada com o auxílio de data show, abordando assuntos como câncer de pele melanoma e não melanoma (conceitos, dados epidemiológicos, sinais e sintomas, tratamento), diagnóstico, fatores de risco que contribuem para o surgimento do câncer de pele, prevenção e sobre a fotoproteção.

Ao final da palestra, foi aberto um período para discussão, onde os alunos puderam fazer questionamentos e comentários a respeito do tema abordado. E para finalizar a intervenção, foi feita uma demonstração pela coordenadora do projeto de como se aplicar adequadamente os protetores solares ao longo do dia.

\section{RESULTADOS E ANÁLISES}

Durante a intervenção com os discentes do primeiro período do curso de Zootecnia foi possível observar o interesse dos mesmos para participar da intervenção, principalmente, quando 
Prevenção ao câncer de pele em discentes do curso de Zootecnia: um relato de experiência

se utilizou a ferramenta Plickers, o qual fazia escaneamento do $Q R$ code dos cartões resposta. Recentemente, foi observado que a popularização da tecnologia estimula a participação dos estudantes em sala de aula, de forma que os professores devem se aproveitar das possibilidades trazidas pelo uso de computadores, tablets e smartphones, tão presentes no cotidiano do aluno. Para isto, no entanto, é necessário que o educador adapte suas práticas pedagógicas de maneira que estas dialoguem com os alunos, aumentando o interesse e o engajamento dos mesmos (RIBAS, 2012; GONZALEZ et al., 2015).

À medida que era realizado o escaneamento dos cartões resposta foi possível identificar que os alunos possuíam conhecimento reduzido sobre o tema. Tal padrão de conhecimento pode ser justificado pelo fato de os mesmos estarem cursando o primeiro período do curso de Zootecnia, etapa na qual são ministradas disciplinas básicas como introdução a zootecnia, metodologia científica, química geral e analítica, anatomia animal, em que não são trabalhados conteúdos relacionados ao tema.

Ao se avaliar o projeto pedagógico e a matriz curricular, disponível no site da instituição, do curso de Zootecnia, foi possível identificar que são inexistentes disciplinas que abordem maneiras de prevenção ao câncer de pele e formas de proteção aos raios UV. Em um estudo realizado no estado do Mato Grosso, com estudantes do curso de Zootecnia, também foi possível identificar que os mesmos não possuem na grade da matriz curricular disciplinas com ênfase em saúde ocupacional, sugerindo-se incluir disciplinas que trabalhem conceitos, legislações, medidas de biossegurança e riscos inerentes à prática profissional durante a graduação, a fim de promover maior conhecimento e ofertar segurança aos graduandos (DE OLIVEIRA, 2015). Desta forma, pode-se perceber que durante o processo de elaboração e estruturação dos projetos pedagógicos dos cursos de Zootecnia, não são inseridas disciplinas curriculares que alertem sobre os riscos da profissão, justamente por não serem itens pontuados na diretriz curricular do curso em questão.

A palestra ministrada teve como foco principal levar informações importantes sobre os efeitos da exposição aos raios solares, bem como a utilização de estruturas de sombreamento, dos equipamentos de proteção e da restrição de exposição ao sol em horários específicos. Após a exposição do tema, houve um período de discussão, no qual os alunos do curso tiveram a oportunidade de perguntar, expor o seu ponto de vista e comentar a respeito do que foi ministrado. Sabe-se que as rodas de discussão produzem conhecimentos coletivos e contextualizados, ao privilegiarem a fala crítica e a escuta sensível, de forma lúdica, não usando nem a escrita, nem a leitura da palavra, mas sim dos modos de vida cotidianos (FREIRE, 1996). 
Prevenção ao câncer de pele em discentes do curso de Zootecnia: um relato de experiência

Um ponto interessante observado nesta intervenção é que um dos alunos presente na sala de aula era albino, sendo assim, acredita-se que, por meio da abordagem realizada, este estudante tenha obtido conhecimento suficiente para adotar medidas preventivas contra o surgimento do câncer ao longo da sua formação acadêmica e profissional.

Sabe-se que a progressão do câncer de pele é uma realidade inevitável se não for prevenida, no entanto, existem estratégias educativas que podem minimizar o surgimento da doença, como por exemplo, o uso diário de protetor solar, evitar exposições prolongadas ao sol, utilizar óculos solares com proteção. Entende-se que o albinismo é um fator genético que é caracterizado por haver uma redução ou ausência do pigmento melanina, devido a uma alteração na enzima tirosinase. No albinismo, a função de barreira natural aos raios ultravioleta (UV) da melanina está comprometida, contribuindo para que as pessoas com albinismo estejam mais vulneráveis a queimaduras solares, lesões de pele e câncer (MOREIRA, 2013).

Vale ressaltar ainda que as atividades desenvolvidas envolvendo ensino, pesquisa e extensão nos cursos auxiliam os envolvidos a expandirem suas habilidades e competências. As atividades extensionistas têm importância fundamental na formação do futuro profissional, pois envolvem ao mesmo tempo alunos, professores, pesquisadores, trabalhadores e a comunidade de forma geral.

\section{CONSIDERAÇÕES FINAIS}

Com a realização desta atividade foi possível vivenciar a criação de vínculos de confiança e fortalecimento na relação entre os profissionais da área da saúde com os estudantes e professores do instituto, permitindo que a acadêmica desenvolvedora do projeto fosse capaz de planejar, organizar e executar atividades educacionais que favoreçam a promoção de saúde, além de gerar conscientização sobre o câncer de pele e a importância da fotoproteção para os estudantes do curso de Zootecnia.

Através desta atividade extensionista, foi possível perceber que a discente do curso de Biomedicina, sentiu-se segura e confiante na realização da intervenção, justamente por ao longo do projeto entrar em contato com o tema que seria abordado, planejar as atividades de modo adequado para o grupo a ser trabalhado, obtendo assim uma experiência acadêmica de extrema relevância, assim como profissional. 
Prevenção ao câncer de pele em discentes do curso de Zootecnia: um relato de experiência

\section{AGRADECIMENTOS}

A todos os alunos e ao coordenador do curso de Zootecnia e a Associação Educativa Evangélica de Ceres - GO.

\section{REFERÊNCIAS}

BRASIL, Instituto Nacional de Câncer: câncer de pele não melanoma. Disponível em: https://www.inca.gov.br/tipos-de-cancer/cancer-de-pele-nao-melanoma. Acesso em: 27. Maio. 2021.

BRASIL, Instituto Nacional de Câncer: Pele melanoma. Disponível em: www.inca.gov.br/tipos-de-cancer/cancer-de-pele-melanoma. Acesso em: 27. Maio. 2021.

BRASIL. Instituto Nacional de Câncer José Alencar Gomes da Silva (INCA). Câncer de pele não melanoma. Disponível em: https://www.inca.gov.br/tipos-de-cancer/cancer-de-pelenao-melanoma. Acesso em: 27. Maio. 2021.

BRASIL, 2020. Câncer de Pele. Sociedade Brasileira de Dermatologia. Disponível em: https://www.sbd.org.br/dermatologia/pele/doencas-e-problemas/cancer-da-pele/64/. Acesso em: 27. Maio. 2021.

COSTA, F. B.; WEBER, M. B. Avaliação dos hábitos de exposição ao sol e de fotoproteção dos universitários da Região Metropolitana de Porto Alegre, RS Evaluation of solar exposure and sun-protection behaviors among university students in the. An Bras Dermatol, v. 79, n. 2, p. 149-155, 2004. Disponível em: http://www.scielo.br/pdf/\%0d/abd/v79n2/20061.pdf. Acesso em: 27. Maio. 2021.

DALCIN, M. M. et al. Câncer de pele em trabalhadores rurais: fotoexposição e orientação quanto a fatores de risco. Research, Society and Development, v. 10, n. 1, p. e15110111594e15110111594, 2021. Disponível em: https://rsdjournal.org/index.php/rsd/article/view/11594/10349. Acesso em: 27. Maio. 2021.

FLOR, J.; DAVOLOS, M. R.; CORREA, M. A. Protetores solares. Quim Nova. 2007;30(1):1538. Disponível em: https://www.redalyc.org/pdf/2655/265553579009.pdf. Acesso em: 27. Maio. 2021.

FREIRE, P. Pedagogia da autonomia: saberes necessários a prática pedagógica. São Paulo: Paz e Terra; 1996. Disponível em: https://www.unifalmg.edu.br/extensao/system/files/anexos/A_RODA_DE_CONVERSA_COMO_REC_SA_SA _DE_DA_FAM_LIA.pdf. Acesso em: 27. Maio. 2021.

GONZALEZ, M. A. et al. Teaching and Learning Physics with Smartphones. International Conference on Multimedia in Physics Teaching and Learning, Munich 9-11/9, 2015. Disponível em: https://www.igi-global.com/chapter/teaching-and-learning-physics-withsmartphones/163560. Acesso em: 27. Maio. 2021. 
Prevenção ao câncer de pele em discentes do curso de Zootecnia: um relato de experiência

MAIER, S. R. O et al. A saúde ocupacional no contexto da formação: um estudo qualitativo com graduandos em zootecnia. Revista Eletrônica Gestão e Saúde, n. 3, p. 2482-2496, 2015. Disponível em: https://dialnet.unirioja.es/servlet/articulo?codigo=5555835. Acesso em: 27. Maio. 2021.

MOHER D, A. D. G.; SCHULZ K. F.; SIMERA I. How to develop a reporting guideline. Guidelines for reporting health research: a user's manual. Chichester: John Wiley \& Sons, Ltd; $2014 . \quad$ p. 14-5. Disponível em: https://onlinelibrary.wiley.com/doi/book/10.1002/9781118715598. Acesso em: 27. Maio. 2021.

MOREIRA, S. C. et al. Associação entre a suscetibilidade à exposição solar e a ocorrência de câncer de pele em albinos. Rev. Ciênc. Méd. Biol. Salvador, v. 12, n. 1, p. 70-74, jan./abr. 2013. Disponível em: https://www.scielosp.org/article/physis/2017.v27n2/319-333/pt/. Acesso em: 27. Maio. 2021.

PESSOA, F. O. A.; LEITE, P. R. S. C.; FAQUINELO, P. Zootecnia Campus - Ceres Bacharelado. Projeto Pedagógicos dos Cursos Superiores, 2017. Disponível em: https://ifgoiano.edu.br/home/images/CER/Doc_cursos/Superior/Zootecnia/Zootecnia_Matri z_2017.pdf. Acesso em: 27. Maio. 2021.

PROLLA, P. A. Síndromes de suscetibilidade hereditária ao câncer de pele. Genética molecular do câncer. São Paulo: MSG Produção Editorial, p. 213-222, 2002. Disponível em: https://www.scielosp.org/scielo.php?pid=S141381232008000400030\&script=sci_arttext\&tlng= es. Acesso em: 27. Maio. 2021.

PURIM, K. S. M. et al. Perfil de Casos de Melanoma em um Hospital Universitário, 2003 a 2007. Rev Bras Cancerol, v. 59, n. 2, p. 193-9, 2013. Disponível em: https://rbc.inca.gov.br/site/arquivos/n_59/v02/pdf/06-perfil-de-casos-de-melanoma-em-umhospital-universitario-2003-a-2007.pdf. Acesso em: 27. Maio. 2021.

RIBAS, A. S. Telefone celular como um recurso didático: possibilidades para mediar práticas do ensino de Física. Dissertação de mestrado. Universidade Tecnológica Federal do Paraná, campus Ponta Grossa-PPGECT, 2012. Disponível em: https://repositorio.utfpr.edu.br/jspui/bitstream/1/1236/1/PG_PPGECT_M_Ribas\%2c\%20Ar ilson\%20Sartorelli_2012.pdf. Acesso em: 27. Maio. 2021.

SANTOS, S. O.; SOBRINHO, R. R.; OLIVEIRA, T. A. Importância do uso de protetor solar na prevenção do câncer de pele e análise das informações desses produtos destinados a seus usuários. Journal of Health \& Biological Sciences, v. 6, n. 3, p. 279-285, 2018. Disponível em: https://periodicos.unichristus.edu.br/jhbs/article/view/1913. Acesso em: 27. Maio. 2021.

SILVA, D. O.; SALES, G. L.; CASTRO, J. B. A Utilização Do Aplicativo Plickers Como Ferramenta $\mathrm{Na}$ Implementação $\mathrm{Da}$ Metodologia Peer Instruction. Revista Eletrônica Científica Ensino Interdisciplinar, v. 4, n. 12, p. 502-516, 2018. Disponível em: http://hdl.handle.net/10400.8/3276. Acesso em: 27. Maio. 2021.

SILVA, J. M.; SILVA, E. M.; FARIA, H. P.; PINHEIRO, T. M. M. Agrotóxico e trabalho: uma combinação perigosa para a saúde do trabalhador rural. Ciênc Saúde coleva. 2005; 10(4):891903. Disponível em: https://www.scielosp.org/article/csc/2005.v10n4/891-903/. Acesso em: 27. Maio. 2021. 
Prevenção ao câncer de pele em discentes do curso de Zootecnia: um relato de experiência

TRICHOPOULOS, D.; LI, J.; HUNTER, D. J. What causes cancer?. Scientific American, 1996. Disponível em: < http://encognitive.com/node/4164>. Acesso em: 27. Maio. 2021.

ZINK, B. S. Câncer de pele: a importância do seu diagnóstico, tratamento e prevenção. Revista HUPE, Rio de Janeiro, v. 13, supl. 1, ago 2014. Disponível em: revista.hupe.uerj.br/audiencia_pdf.asp?aid2 $=480 \&$ nomeArquivo $=v 13$ s1a11.pdf. Acesso em: 27. Maio. 2021.

Recebido em: 07/02/2020

Aceito em: 15/04/2021 